\title{
The Nuclear Effect Induced by Additional Parton Evo- lution and Recombination in Nuclear Environment
}

\author{
Jian-Jun Yang ${ }^{1,3}$, Guang-Lie Li ${ }^{2,3}$ \\ ${ }^{1}$ Department of Physics, Nanjing Normal University, Nanjing 210024, China \\ ${ }^{2}$ CCAST(World Laboratory), P.O.Box 8730, Beijing 100080, China \\ ${ }^{3}$ Institute of High Energy Physics, Academia Sinica, P.O.Box 918(4), Beijing \\ 100039, China円
}

\begin{abstract}
The relationship between the parton transverse momentum and longitudinal momentum, which is obtained by analysing the hadron tensor in the deep inelastic scattering (DIS) process, indicates that the transverse size of partons in a nucleon is sensitively affected by the effective nucleon mass. The change in this transverse size gives rise to an Additional Parton Evolution (APE) in the nuclear environment refer to the normal QCD parton evolution thus resulting in new parton distributions. The self-consistent integral equations for these new parton distributions are given. Having undergone the normal QCD evolution and the additional evolution in the nuclear environment, the partons with the small $x$ participate in recombination and contribute to the nuclear shadowing effect. The reasonably good descriptions of the nuclear effect of structure functions, nuclear Drell-Yan ratios and nuclear gluon distributions are presented. A quantitative comparison between the APE model and the $Q^{2}$-rescaling model is made.
\end{abstract}

\footnotetext{
${ }^{1}$ Mailing address. Email address: yangjj@bepc3.ihep.ac.cn
} 


\section{Introduction}

Since the discovery of the nuclear modification in the nucleon structure function $F_{2}\left(x, Q^{2}\right)$ by the European Muon Collaboration (EMC) [1], various models have been proposed to investigate the nuclear effect. Several years ago, the New Muon Collaboration (NMC) [2] accurately measured the structure functions $F_{2}$ at very small $x$. These data as well as the EMC data [3] provide an insight for investigating the physics details in the shadowing region $(x<0.1)$. The need for testing the models of the EMC effect becomes more and more important for further understanding of the nature of the nuclear effect. Bickerstaff et al. [1] found that although most of the theoretical models can provide good explanations for the EMC effect, they can not describe the nuclear Drell-Yan process well. The NMC [5] analyzed the inelastic $J / \psi$ production data and obtained the gluon distribution ratios $G_{S n}(x) / G_{C}(x)$ which indicate a modification of the gluon distribution in the nuclear environment. Kumano [6] has made progress towards the unified description of the EMC effect and the nuclear gluon distribution in the $Q^{2}$-rescaling model with the recombination effect. Although the $Q^{2}$-rescaling model is a useful and effective model in explaining deep-inelastic data in the valence dominated medium $x$ region, it is not clear whether the momentum distributions of sea quarks and gluons follow the same rescaling as that for valence quarks in the nuclear environment. According to the QCD theory, sea quarks have different dynamic properties while interacting with gluons. There is a constraint condition on the number conservation for valence quarks but not for sea quarks. So it is reasonable to believe that the modification of sea quark distributions should be different from that of valence quarks. For further discussion, we assume that the transverse size of partons in a nucleon can be described by an average parton transverse momentum which is related to the longitudinal distributions of partons. The relationship between the parton transverse momentum and longitudinal momentum, which is obtained by analysing the hadron tensor in the deep inelastic scattering process, indi-

cates that the transverse size of partons in a nucleon depends sensitively on the effective nucleon mass. The change of this transverse size gives rise to an additional parton evolution in the nuclear environment refer to the normal QCD parton evolution thus resulting in new parton distributions. The probabilities of the additional evolution are directly related to the ratio of the average parton transverse momentum in the bound nucleon to that in 
the free nucleon. According to these probabilities, we give the self-consistent integral equations for parton distribution modifications due to the additional evolution in the nuclear environment.

Considering the fact that the nucleon diameter is about $1.8 \mathrm{fm}$, in an infinite momentum frame, one can estimate that the longitudinal size of a nucleon in a Lorentz contracted nucleus is

$$
D=1.8(\mathrm{fm}) m_{N} / p_{N}
$$

and the longitudinal localization size of a parton with the momentum $x p_{N}$ is $L=1 /\left(x p_{N}\right)$. If a parton has a small $x$ and consequently its dimension $(L)$ exceeds the nucleon longitudinal size $(D)$, it leaks out of the nucleon and recombines with partons of other nucleons. We suppose that the partons participating in recombination have undergone the normal QCD evolution and the additional evolution in the nuclear environment before the fusion. After considering the additional evolution in the nuclear environment with the parton recombination effects, we find that the new model can give a well explaination to the nuclear effects of the structure function, nuclear DrellYan process and inelastic $J / \psi$ production. Finally, a quantitative comparison between the APE model and the $Q^{2}$-rescaling model is made.

\section{Additional Parton Evolution(APE) Due to Nuclear Environment}

Partons in a free nucleon have their own distributions with a specified scale. It is generally accepted that the transverse size of partons in a nucleon is affected by the nuclear environment. We introduce an average transverse momentum to describe the change of the transverse size of partons.

In order to obtain the average transverse momentum of partons, let us repeat the $\xi$ variable analysis [8] of the hadron tensor $W_{\mu \nu}$ in the deep inelastic scattering process shown in Fig.1. $W_{\mu \nu}$ can be written as

$$
\begin{aligned}
W_{\mu \nu} & =\frac{1}{2 \pi} \int d^{4} x e^{i q \cdot x}\left\langle p\left|\left[\mathbf{J}_{\mu}(x), \mathbf{J}_{\nu}(0)\right]\right| p\right\rangle \\
& =\left(-g_{\mu \nu}+\frac{q_{\mu} q_{\nu}}{q^{2}}\right) W_{1}+\frac{1}{m_{N}^{2}}\left(p_{\mu}-\frac{p \cdot q}{q^{2}} q_{\mu}\right)\left(p_{\nu}-\frac{p \cdot q}{q^{2}} q_{\nu}\right) W_{2}
\end{aligned}
$$


where $m_{N}$ is the mass of a nucleon. If we take the frame in which $p=\left(m_{N}, \mathbf{0}\right)$ and neglect the quark mass, then

$$
W_{\mu \nu} \propto \int \frac{d^{3} k}{2 k_{0}} f\left(\frac{2 k_{0}}{m_{N}}\right) \delta\left(q^{2}+2 k \cdot q\right) w_{\mu \nu}
$$

where

$$
w_{\mu \nu}=\left[(k+q)_{\mu} k_{\nu}+(k+q)_{\nu} k_{\mu}+\frac{q^{2}}{2} g_{\mu \nu}\right]
$$

is the quark tensor in the lab. frame and $f(k \cdot p)$ is a Lorentz-invariant wave function. To extract $W_{1}$ and $W_{2}$ from $W_{\mu \nu}$, we introduce two contractions

$$
\begin{gathered}
B=g^{\mu \nu} W_{\mu \nu}=-3 W_{1}+\left(1+\frac{\nu^{2}}{Q^{2}}\right) W_{2} \\
C=\frac{1}{m_{N}^{2}\left(1+\nu^{2} / Q^{2}\right)} p^{\mu} p^{\nu} W_{\mu \nu}=-W_{1}+\left(1+\frac{\nu^{2}}{Q^{2}}\right) W_{2}
\end{gathered}
$$

and then $W_{1}$ and $W_{2}$ can be expressed as

$$
\begin{gathered}
W_{1}=\frac{1}{2}(C-B) \\
W_{2}=\frac{3 C-B}{2\left(1+\nu^{2} / Q^{2}\right)}
\end{gathered}
$$

If we take the frame in which $q=\left(\nu, 0,0, \sqrt{\nu^{2}+Q^{2}}\right)$ and $k=\left(k_{0}, 0, k_{0} \sin \theta, k_{0} \cos \theta\right)$, then

$$
k_{\perp}^{2}=k_{0}^{2} \sin ^{2} \theta=\frac{2 k_{0}^{2}+2 k_{0} \nu-\frac{1}{2} Q^{2}}{2\left(1+\nu^{2} / Q^{2}\right)}
$$

By means of Eq.(22) and the contractions defined by Eq.(田) and Eq.(5), one can find the relation

$$
\left\langle k_{\perp}^{2}\right\rangle=-\frac{C Q^{2}}{2 B}
$$

Taking $W_{1}$ and $W_{2}$ in Eq.(4) and Eq.(5) as those in Refs. [9] and 10 and neglecting $O\left(m_{N}^{2} / Q^{2}\right)$ and $O\left(Q^{2} / \nu^{2}\right)$ terms, one easily obtains

$$
\left\langle K_{\perp}^{2}\left(x, Q^{2}\right)\right\rangle^{q^{N(A)}}=\frac{x^{3} m_{N(A)}^{2}}{x q^{N(A)}\left(x, Q^{2}\right)} \int_{x}^{1} d x^{\prime} \frac{x^{\prime} q^{N(A)}\left(x^{\prime}, Q^{2}\right)}{x^{\prime 2}}
$$


Furthermore, we assume that there is a similar relationship between the gluon transverse momentum and longitudinal momentum distributions, i.e.,

$$
\left\langle K_{\perp}^{2}\left(x, Q^{2}\right)\right\rangle^{G^{N(A)}}=\frac{x^{3} m_{N(A)}^{2}}{x G^{N(A)}\left(x, Q^{2}\right)} \int_{x}^{1} d x^{\prime} \frac{x^{\prime} G^{N(A)}\left(x^{\prime}, Q^{2}\right)}{x^{\prime 2}}
$$

Eq.(10) and Eq.(11) with the bound nucleon effective mass $m_{N(A)}\left(m_{N(1)}=\right.$ $m_{N}$ ) have been written in a unified form for partons in both a free nucleon(N) and a bound nucleon(A) in a nucleus with the mass number $A$. The effective mass of the bound nucleon is less than that of the free one, i.e., in terms of Eq.(10) and Eq.(11), the average transverse momentum of partons in the bound nucleon is less than that in the free nucleon, which mainly causes the parton evolution in the nuclear environment. By using the same technique in deriving the famous Altarelli-Parisi evolution [11], we obtain the evolution probabilities for the evolution from a parton with momentum fraction $y$ to another parton with the momentum fraction $x$;

$$
\begin{gathered}
F_{q_{i} \rightarrow q_{j}}\left(\frac{x}{y}\right)=\frac{\alpha_{s}}{2 \pi} C_{2}(R)\left[\frac{1+\left(\frac{x}{y}\right)^{2}}{\left(1-\frac{x}{y}\right)_{+}}+\frac{3}{2} \delta\left(1-\frac{x}{y}\right)\right] \ln \frac{\left\langle K_{\perp}^{2}\left(x, Q^{2}\right)\right\rangle^{q_{j}^{N}}}{\left\langle K_{\perp}^{2}\left(x, Q^{2}\right)\right\rangle^{q_{j}^{A}}} \\
F_{G \rightarrow q_{j}}\left(\frac{x}{y}\right)=\frac{\alpha_{s}}{4 \pi}\left[\left(\frac{x}{y}\right)^{2}+\left(1-\frac{x}{y}\right)^{2}\right] \ln \frac{\left\langle K_{\perp}^{2}\left(x, Q^{2}\right)\right\rangle^{q_{j}^{N}}}{\left\langle K_{\perp}^{2}\left(x, Q^{2}\right)\right\rangle^{q_{j}^{A}}} \\
F_{q_{i} \rightarrow G}\left(\frac{x}{y}\right)=\frac{\alpha_{s}}{2 \pi} C_{2}(R)\left[\frac{1+\left(1-\frac{x}{y}\right)^{2}}{\frac{x}{y}}\right] \ln \frac{\left\langle K_{\perp}^{2}\left(x, Q^{2}\right)\right\rangle^{G^{N}}}{\left\langle K_{\perp}^{2}\left(x, Q^{2}\right)\right\rangle^{G^{A}}} \\
F_{G \rightarrow G}\left(\frac{x}{y}\right)=\frac{\alpha_{s}}{\pi} C_{2}(G)\left[\frac{\frac{x}{y}}{\left(1-\frac{x}{y}\right)_{+}}+\frac{\left(1-\frac{x}{y}\right)}{\frac{x}{y}}+\frac{x}{y}\left(1-\frac{x}{y}\right)\right. \\
\left.+\frac{1}{12}\left(11-\frac{2 N_{f}}{3}\right) \delta\left(1-\frac{x}{y}\right)\right] \ln \frac{\left\langle K_{\perp}^{2}\left(x, Q^{2}\right)\right\rangle^{G^{N}}}{\left\langle K_{\perp}^{2}\left(x, Q^{2}\right)\right\rangle^{G^{A}}}
\end{gathered}
$$

where the strong interaction coupling constant $\alpha_{s}$ is

$$
\alpha_{s}\left(Q^{2}\right)=4 \pi /\left[\beta_{0} \ln \left(Q^{2} / \Lambda^{2}\right)\right]
$$

with $\Lambda=0.19 G e V$ and $\beta_{0}=11-2 N_{f} / 3, N_{f}=3 . C_{2}(R)=\frac{3}{4}$ and $C_{2}(G)=3$ are color factors for $N_{c}=3$. Therefore, the quark distributions modified 
by the additional parton evolution(APE) in the nuclear environment can be written as follows:

$$
\begin{aligned}
q_{v}^{A P E}\left(x, Q^{2}\right) & =q_{v}^{N}\left(x, Q^{2}\right)+\int_{x}^{1} F_{q_{v} \rightarrow q_{v}}\left(\frac{x}{y}\right) \frac{q_{v}^{N}\left(y, Q^{2}\right)}{y} d y \\
q_{s}^{A P E}\left(x, Q^{2}\right) & =q_{s}^{N}\left(x, Q^{2}\right)+\int_{x}^{1} F_{q_{s} \rightarrow q_{s}}\left(\frac{x}{y}\right) \frac{q_{s}^{N}\left(y, Q^{2}\right)}{y} d y \\
& +\int_{x}^{1} F_{G \rightarrow q_{s}}\left(\frac{x}{y}\right) \frac{G^{N}\left(y, Q^{2}\right)}{y} d y \\
G^{A P E}\left(x, Q^{2}\right)= & G^{N}\left(x, Q^{2}\right)+\sum_{i} \int_{x}^{1} F_{q_{i} \rightarrow G}\left(\frac{x}{y}\right) \frac{q_{i}^{N}\left(y, Q^{2}\right)}{y} d y \\
& +\int_{x}^{1} F_{G \rightarrow G}\left(\frac{x}{y}\right) \frac{G^{N}\left(y, Q^{2}\right)}{y} d y
\end{aligned}
$$

where the input parton distributions $q^{N}\left(x, Q^{2}\right)$ and $G^{N}\left(x, Q^{2}\right)$ are taken from Refs. [12]- [14]. In fact, these equations are the self-consistent integral equations since the evolution probabilities $F_{p_{i} \rightarrow p_{j}}(z)$ contain the parton transverse momenta which are related to the parton longitudinal momentum distributions $q^{N(A)}\left(x, Q^{2}\right)$ and $G^{N(A)}\left(x, Q^{2}\right)$ by Eq.(10) and Eq.(11). In the calculation of the parton distributions in the bound nucleon, the numerical solution of these equations is obtained by the iteration method.

\section{Leak-out Sea Quarks and Gluons in Nu- clear Environment}

Although the leakage in the nuclear environment can occur for all partons, the most important contribution arises from partons with the largest spatial uncertainty, i.e., those with the small $x$. We assume that the leak-out partons are sea quarks and gluons for which the momentum cutoff function [15] is taken as

$$
\beta(x)=\exp \left(-\frac{m_{N}^{2} y_{0}^{2} x^{2}}{2}\right),
$$

namely, the distributions of leak-out(LK) partons are

$$
p^{L K}\left(x, Q_{0}^{2}\right)=\beta(x) p^{A P E}\left(x, Q_{0}^{2}\right)
$$


where the input parton distributions $p^{A P E}=u_{s}^{A P E}, \bar{u}^{A P E}, d_{s}^{A P E}, \bar{d}^{A P E}, s^{A P E}$

, $\bar{s}^{A P E}$ and $G^{A P E}$ together with $u_{v}^{A P E}$ and $d_{v}^{A P E}$ are those having undergone the normal QCD parton evolution and the additional parton evolution due to the nuclear environment. The momentum cutoff function is shown as a function of $x$ in Fig. 2 with various $y_{0}$. As discussed in section 1 , the partons with $x \leq 0.11$ can leak out of a nucleon. We then find in Fig.2 that an appropriate choice of $y_{0}$ is $2.0 \mathrm{fm}$.

\section{Parton Recombination and Further Mod- ification to Parton Distributions}

According to the original idea of the parton recombination by Close, Qiu and Roberts 15, a leak-out parton can fuse with a parton from another nucleon. In general, the modification of a parton distribution $p_{3}\left(x_{3}\right)$, due to the process of producing the parton $p_{3}$ with the momentum $x_{3}$ by fusion of partons $p_{1}$ and $p_{2}$, is given by [15],

$$
\begin{gathered}
\Delta p_{3}\left(x_{3}\right)=K \int d x_{1} d x_{2} p_{1}\left(x_{1}\right) p_{2}\left(x_{2}\right) \\
\times \Gamma_{p_{1} p_{2} \rightarrow p_{3}}\left(x_{1}, x_{2}, x_{3}=x_{1}+x_{2}\right) \delta\left(x_{3}, x_{1}, x_{2}\right),
\end{gathered}
$$

where $K$ is given by Refs. [15 and [16],

$$
K=9 A^{1 / 3} \alpha_{s} /\left(2 R_{0}^{2} Q_{0}^{2}\right)
$$

The nuclear radius is $R=R_{0} A^{1 / 3}$ with $R_{0}=1.1 \mathrm{fm}$ [17]. The function $\delta\left(x_{3}, x_{1}, x_{2}\right)$ defined by Ref. [6] is introduced to keep the momentum conservation. The parton fusion function $\Gamma_{p_{1} p_{2} \rightarrow p_{3}}\left(x_{1}, x_{2}, x_{3}\right)$ is a probability for producing a parton $p_{3}$ with momentum $x_{3}$ by a fusion of partons $p_{1}$ and $p_{2}$ with momentum $x_{1}$ and $x_{2}$, respectively. It is related to a splitting function $P_{p_{1} \leftarrow p_{3}}(z)$ in the Altarelli-Parisi equations [11] by

$$
\Gamma_{p_{1} p_{2} \rightarrow p_{3}}\left(x_{1}, x_{2}, x_{3}\right)=\frac{x_{1} x_{2}}{x_{3}^{2}} P_{p_{1} \leftarrow p_{3}}\left[\frac{x_{1}}{x_{3}}\right] C_{p_{1} p_{2} \rightarrow p_{3}} .
$$

where $C_{p_{1} p_{2} \rightarrow p_{3}}$ is the ratio of color factors in the processe $p_{1} p_{2} \rightarrow p_{3}$. Using the fusion functions, one can obtain the modifications of parton distributions 
due to the recombination $(\mathrm{RC})[6]$. With our notations, the explicit expressions for the modifications $\Delta q^{R C}(x)$ and $\Delta G^{R C}(x)$ are

$$
\begin{aligned}
x \Delta & q^{R C}(x) \\
= & \frac{K}{6} \int_{0}^{x} \frac{d x^{\prime}}{x^{\prime}} x^{\prime}\left(x-x^{\prime}\right)\left[G^{L K}\left(x^{\prime}\right) q^{A P E}\left(x-x^{\prime}\right)\right. \\
& \left.+G^{A P E}\left(x^{\prime}\right) q^{L K}\left(x-x^{\prime}\right)\right]\left[1+\left[\frac{x-x^{\prime}}{x}\right]\right] \\
& -\frac{K}{6} \int_{0}^{1} \frac{d x^{\prime}}{x^{\prime}} x x^{\prime}\left[q^{A P E}(x) G^{L K}\left(x^{\prime}\right)+q^{L K}(x) G^{A P E}\left(x^{\prime}\right)\right] \frac{x}{x+x^{\prime}}\left[1+\left[\frac{x}{x+x^{\prime}}\right]^{2}\right] \\
& -\frac{4 K}{9} x \int_{0}^{1} d x^{\prime} x x^{\prime}\left[q^{A P E}(x) \bar{q}^{L K}\left(x^{\prime}\right)+q^{L K}(x) \bar{q}^{A P E}\left(x^{\prime}\right)\right] \frac{x^{2}+x^{\prime 2}}{\left(x+x^{\prime}\right)^{4}}
\end{aligned}
$$

and

$$
\begin{aligned}
x \Delta & G^{R C}(x) \\
= & \frac{3 K}{4} x \int_{0}^{x} d x^{\prime} x^{\prime}\left(x-x^{\prime}\right)\left[G^{A P E}\left(x^{\prime}\right) G^{L K}\left(x-x^{\prime}\right)\right. \\
& \left.+G^{L K}\left(x^{\prime}\right) G^{A P E}\left(x-x^{\prime}\right)\right] \frac{1}{x^{2}}\left[\frac{x^{\prime}}{x-x^{\prime}}+\frac{x-x^{\prime}}{x^{\prime}}+\frac{x^{\prime}\left(x-x^{\prime}\right)}{x^{2}}\right] \\
& -\frac{3 K}{4} x \int_{0}^{1} d x^{\prime} x x^{\prime}\left[G^{L K}(x) G^{A P E}\left(x^{\prime}\right)+G^{A P E}(x) G^{L K}\left(x^{\prime}\right)\right] \\
& \times \frac{1}{\left(x+x^{\prime}\right)^{2}}\left[\frac{x}{x^{\prime}}+\frac{x^{\prime}}{x}+\frac{x x^{\prime}}{\left(x+x^{\prime}\right)^{2}}\right] \\
& +\frac{4 K}{9} x \int_{0}^{x} d x^{\prime} \sum_{i}\left[x^{\prime} q_{i}^{A P E}\left(x^{\prime}\right)\left(x-x^{\prime}\right) \bar{q}_{i}^{L K}\left(x-x^{\prime}\right)\right. \\
& \left.+x^{\prime} \bar{q}_{i}^{A P E}\left(x^{\prime}\right)\left(x-x^{\prime}\right) q_{i}^{L K}\left(x-x^{\prime}\right)\right] \frac{1}{x^{4}}\left[x^{\prime 2}+\left(x-x^{\prime}\right)^{2}\right] \\
& -\frac{K}{6} \int_{0}^{1} d x^{\prime} x G^{A P E}(x) \sum_{i}\left[x^{\prime} q_{i}^{L K}\left(x^{\prime}\right)+x^{\prime} \bar{q}_{i}^{L K}\left(x^{\prime}\right)\right] \frac{1}{x+x^{\prime}}\left[1+\left[\frac{x^{\prime}}{x+x^{\prime}}\right]^{2}\right] \\
& -\frac{K}{6} \int_{0}^{1} d x^{\prime} x G^{L K}(x) \sum_{i}\left[x^{\prime} q_{i}^{A P E}\left(x^{\prime}\right)+x^{\prime} \bar{q}_{i}^{A P E}\left(x^{\prime}\right)\right] \\
& \times \frac{1}{x+x^{\prime}}\left[1+\left[\frac{x^{\prime}}{x+x^{\prime}}\right]\right]
\end{aligned}
$$

The explicit $Q^{2}$ dependence in the parton distributions is not shown in the above two equations in order to simplify the notation. Therefore, we write, 
for the nuclear gluon distribution,

$$
x G^{A}\left(x, Q_{0}^{2}\right)=x G^{A P E}\left(x, Q_{0}^{2}\right)+\Delta x G^{R C}\left(x, Q_{0}^{2}\right)
$$

Similarly, for the nuclear quark distribution,

$$
x q^{A}\left(x, Q_{0}^{2}\right)=x q^{A P E}\left(x, Q_{0}^{2}\right)+\Delta x q^{R C}\left(x, Q_{0}^{2}\right)+\Delta x q_{s}^{G S}\left(x, Q_{0}^{2}\right)
$$

where $\Delta x q_{s}^{G S}\left(x, Q_{0}^{2}\right)$ is the modification due to the gluon shadowing(GS) and is derived from the evolution equations. A crude estimate of $\Delta x q_{s}^{G S}\left(x, Q_{0}^{2}\right)$ is given by 15

$$
x q_{s}\left(x, Q_{0}^{2}\right)=-\frac{x}{12} \frac{\partial\left[x G\left(x, Q_{0}^{2}\right)\right]}{\partial x}
$$

Using this relation, we expect that the sea-quark distribution is also affected by the gluon modification as follows:

$$
x \Delta q_{s}^{G S}\left(x, Q_{0}^{2}\right)=-\theta\left(x_{0}-x\right) \frac{x}{12} \frac{\partial\left[x \Delta G^{R C}\left(x, Q_{0}^{2}\right)\right]}{\partial x}
$$

where the step function $\theta\left(x_{0}-x\right)$ is introduced because the relation is valid only at very small $x$ ( $x$ less than $x_{0}$ with $\left.x_{0}=0.1\right)$.

\section{Explanation of Nuclear Effect in Nucleon Parton Distributions}

We have considered the additional parton evolution and parton recombination in sections 2 and 4, respectively, and gotten the nuclear parton distributions at $Q_{0}^{2}=4.0 \mathrm{GeV}^{2}$. To compare the results with the experimental data or display the $Q^{2}$-dependence of the model, these distributions are evolved to those at lager $Q^{2}$ by using the ordinary Altarelli-Parisi equations [11]. For the valence quark $\left(q_{v}\right)$, sea quark $\left(q_{s}\right)$, and $\operatorname{gluon}(\mathrm{G})$, the evolution equations are

$$
\begin{gathered}
\frac{\partial}{\partial t} q_{v}^{A}(x, t)=\int_{x}^{1} \frac{d y}{y} q_{v}^{A}(y, t) P_{q q}\left[\frac{x}{y}\right] \\
\frac{\partial}{\partial t} q_{s}^{A}(x, t)=\int_{x}^{1} \frac{d y}{y}\left[q_{s}^{A}(y, t) P_{q q}\left[\frac{x}{y}\right]+G^{A}(y, t) P_{q G}\left[\frac{x}{y}\right]\right]
\end{gathered}
$$




$$
\frac{\partial}{\partial t} G^{A}(x, t)=\int_{x}^{1} \frac{d y}{y}\left[q_{s}^{A}(y, t) P_{G q}\left[\frac{x}{y}\right]+G^{A}(y, t) P_{G G}\left[\frac{x}{y}\right]\right]
$$

where $P_{i j}$ is the splitting function, and $t$ is defined by

$$
t=-\left(2 / \beta_{0}\right) \ln \left[\alpha_{s}\left(Q^{2}\right) / \alpha_{s}\left(Q_{0}^{2}\right)\right]
$$

We used the solution of these integral equations in the leading order and the evolution subroutine of Ref. [18] in our numerical calculation.

\subsection{Nuclear Structure Function $F_{2}^{A}(x)$}

We now proceed to compute the modification in the structure function. In order to compare with the experimental data, let us define the ratio of the average nuclear structure function to the deuteron structure function as [19]

$$
R^{A / D}\left(x, Q^{2}\right)=\frac{F_{2}^{A}\left(x, Q^{2}\right)}{F_{2}^{D}\left(x, Q^{2}\right)}
$$

with the average nuclear structure function:

$$
F_{2}^{A}\left(x, Q^{2}\right)=\frac{1}{A}\left[F_{2 A}\left(x, Q^{2}\right)-\frac{1}{2}(N-Z)\left(F_{2}^{n}\left(x, Q^{2}\right)-F_{2}^{p}\left(x, Q^{2}\right)\right)\right]
$$

where $F_{2}^{n}$ and $F_{2}^{p}$ are the free neutron and proton structure functions, respectively. $F_{2 A}\left(x, Q^{2}\right)$ is the nuclear structure function. The second term compensates for the neutron excess. By considering the Fermi motion of nucleons in the nucleus, $F_{2 A}\left(x, Q^{2}\right)$ can be written as [19]

$$
F_{2 A}\left(x, Q^{2}\right)=\sum_{\lambda} \int \frac{d^{3} p}{(2 \pi)^{3}}\left|\psi_{\lambda}(\vec{p})\right|^{2} z F_{2}^{N(A)}\left(\frac{x}{z}, Q^{2}\right),
$$

with $z=\left(p_{0}+p_{3}\right) / m_{N}, p_{0}=m_{N}+\epsilon_{\lambda}, \epsilon_{\lambda}$ is the separation energy of a nucleon in the single-particle state $\lambda$, and $\psi_{\lambda}(\vec{p})$ is the single-particle wave function of the nucleon in the momentum space, which satisfies the light-cone normalization:

$$
\int \frac{d^{3} p}{(2 \pi)^{3}}\left|\psi_{\lambda}(\vec{p})\right|^{2} z=1
$$

In the following calculation, $\epsilon_{\lambda}$ and $\psi_{\lambda}(\vec{p})$ are taken from Ref. 20. By using the nuclear parton distributions after considering the additional parton 
evolution, recombination and the ordinary Altarelli-Parisi evolution to higher $Q^{2}$, the bound nucleon structure function $F_{2}^{N(A)}\left(x, Q^{2}\right)$ can be expressed as

$$
\begin{gathered}
F_{2}^{N(A)}\left(x, Q^{2}\right) \\
=\frac{1}{18} x\left\{5 \left[u_{v}^{A}\left(x, Q^{2}\right)+d_{v}^{A}\left(x, Q^{2}\right)+u_{s}^{A}\left(x, Q^{2}\right)+\bar{u}^{A}\left(x, Q^{2}\right)+d_{s}^{A}\left(x, Q^{2}\right)\right.\right. \\
\left.\left.+\bar{d}^{A}\left(x, Q^{2}\right)\right]+2\left[s^{A}\left(x, Q^{2}\right)+\bar{s}^{A}\left(x, Q^{2}\right)\right]\right\}
\end{gathered}
$$

We now calculate $R^{A / D}\left(x, Q^{2}\right)$ in Eq.(30). The deuteron structure function $F_{2}^{D}\left(x, Q^{2}\right)$ is taken from Ref. 21]. Similar to Ref. 222, we take

$$
m_{N(A)}=\frac{1}{1+0.48 \ln \left(2-A^{-1 / 3}\right]}
$$

where the constant 0.48 is determined by $m_{N(\infty)}=0.75 m_{N}$ [23.

The theoretical result in the range $0.1<x<0.2$ can not be well described since the gluonic modification due to Eq. (24) is valid at very small $x$. However, using the KMRS-B0]12], our calculation results indicate that the modified parton distribution can be well parametrized by the analytical form of the corresponding input distribution. The parameters in the analytical form of the modified parton distributions can be obtained by fitting the numerical results. So we obtain a smooth curve of the ratio $R^{C a / D}\left(x, Q^{2}\right)$ which is compared with the experimental data of the EMC effect [1] in Fig.3. The result is in qualitative agreement with the experimental data. To show the distinct effect of the additional parton evolution, the following results are no longer presented by fitting numerical results and taking into account the Fermi-motion correction.

In order to investigate the $Q^{2}$-dependence of the ratio $R^{C a / D}\left(x, Q^{2}\right)$, the results obtained by using the GRV inputs 13 and the KMRS-B0 inputs 12 are plotted in Fig.4(a) and Fig.4(b), respectively. One can easily find that the result obtained by using the KMRS-B0 inputs is less dependent on $Q^{2}$. In Fig.5, the results with various input parton distributions of different parameterizations are presented, and they show that, in the small $x$ region, the recombination results after undergoing the additional parton evolution are very sensitive to the input sea-quark and gluon distributions. Using the Duke-Owens(2) inputs of Ref. [14] with hard gluon distributions, theoretical 
results underestimate the shadowing, which is not difficult to be understood since the parton parameterizations of Ref. [14] did not fit to the experimental data in the small $x$ region. This is the reason why we take KMRS-B0 [12 or GRV [13 parameterizations as input parton distributions in most of the following calculations.

\subsection{Nuclear Drell-Yan Ratio}

Several years ago, the E772 Collaboration [24] at Fermilab published the data of high-mass dilepton production measured in the nuclear Drell-Yan (DY) process. These data aroused special attention in clarifying the different explanations for the nuclear effect on the parton distributions. Their results show that the ratio of DY dimuon yield per nucleon on a nuclear target to that on a free nucleon is slightly less than unity if the momentum fraction $x$ carried by a target quark is less than 0.1 . The ratios over the range $0.1<x<0.3$, however, do not reveal distinct nuclear dependence.

In order to show the prediction of nuclear Drell-Yan ratio in our present model, let us begin with the definition of the nuclear Drell-Yan ratio :

$$
T^{A / D}(x)=\frac{\int d x^{\prime} d^{2} \sigma^{h-A}\left(x^{\prime}, x\right) / d x^{\prime} d x}{\int d x^{\prime} d^{2} \sigma^{h-D}\left(x^{\prime}, x\right) / d x^{\prime} d x},
$$

where the differential cross section of the nuclear Drell-Yan process is:

$$
\frac{d^{2} \sigma^{h-A}}{d x^{\prime} d x}=\frac{1}{3} \frac{4 \pi \alpha^{2}}{3 x^{\prime} x M^{2}} H_{h-A}\left(x^{\prime}, x\right)
$$

with

$$
H_{h-A}\left(x^{\prime}, x\right)=\sum_{i} e_{i}^{2}\left[x^{\prime} q_{i}^{h}\left(x^{\prime}\right) x \bar{q}_{i}^{A}(x)+x^{\prime} \bar{q}_{i}^{h}\left(x^{\prime}\right) x q_{i}^{A}(x)\right]
$$

In a more specified case for the proton-nucleus reaction:

$$
\begin{gathered}
H_{p-A}\left(x^{\prime}, x, Q^{2}\right)=\frac{1}{54} x^{\prime}\left[4 u_{v}^{N}\left(x^{\prime}, Q^{2}\right)+d_{v}^{N}\left(x^{\prime}, Q^{2}\right)\right] x S^{A}\left(x, Q^{2}\right) \\
+\frac{1}{54 A} x^{\prime} S^{N}\left(x^{\prime}, Q^{2}\right) x\left[(A+3 Z) u_{v}^{A}\left(x, Q^{2}\right)\right. \\
\left.+(4 A-3 Z) d_{v}^{A}\left(x, Q^{2}\right)+2 A S^{A}\left(x, Q^{2}\right)\right]
\end{gathered}
$$


where the total sea quark is $S=u_{s}+\bar{u}_{s}+d_{s}+\bar{d}_{s}+s+\bar{s}$. By using the nuclear parton distributions in our model, we calculated the nuclear Drell-Yan ratios for ${ }^{40} \mathrm{Ca}$. The integral range for $x^{\prime}$ in Eq. (36) is determined according to the kinematic region of the experiment in Ref. [24], i.e. $x^{\prime}-x>0$, and $0.025 \leq x \leq 0.30$. To avoid the uncertainty derived by the crude estimate in Eq. (24) for taking into account the gluonic modification, the results, except those in the range $0.1<x<0.2$, are compared with the E772 [24] data in Fig.6. Fig.6 indicates that the APE model as well as the $Q^{2}$-rescaling model can explain the Drell-Yan ratio reasonably well. However, in the small $x$ region $(x<0.1)$, the result obtained by the APE model seems to be better than that of the $Q^{2}$-rescaling model.

\subsection{Nuclear Gluon Distribution}

In 1992, the NMC [5] analyzed the inelastic $J / \psi$ production result with $R_{\text {in }}(S n / C)=1.13 \pm 0.08$ by the color singlet(CS) model 25] and obtained the gluon distribution ratios $G_{S n}(x) / G_{C}(x)$. The result sheds light on gluon distributions in nuclei.

The results of $G_{S n}(x) / G_{C}(x)$ calculated in our model together with the experimental data from NMC are presented in Fig.7, which are qualitatively similar to those given by the $Q^{2}$-rescaling model in Ref. [6]. Due to the gluon shadowing, the ratios $G_{S n}(x) / G_{C}(x)$ are less than 1 at $x<0.09$. It is noteworthy that experimental errors are very large in comparison with typical theoretical modifications. In order to test our model, we should wait for more accurate measurements of $G_{A}(x)$, for example, a proposed experiment at RHIC [26] in the small $x$ region for investigating details of the gluon shadowing.

\section{Discussion and Summary}

It is worthwhile to compare the results of the present model with those of the $Q^{2}$-rescaling model. Although these two models seem to have the same physics origin, they have different descriptions of the nuclear effect on sea quarks and gluons. In the APE model, the evolution of valence quarks, sea quarks and gluons are determined by different interaction vertex factors, and their effects on their own momentum distributions are also different. 
However, the $Q^{2}$-rescaling model, ignoring the difference in additional parton evolution modes for different kinds of partons, describes the influence of the nuclear environment on the distribution functions of valence and sea quarks by using the same $Q^{2}$-rescaling mechanism. In the medium $x$ region where the valence quarks dominate, the $Q^{2}$-rescaling model can explain the EMC effect reasonably well, indicating that the nuclear effect on the valence quark momentum distribution can indeed be well described by the $Q^{2}$-rescaling mechanism. In the small $x$ region where sea quarks and gluons dominate, it is not clear whether the $Q^{2}$-rescaling picture could be used also for sea quarks and gluons. To avoid this uncertainty, we attempt to find a unified description of the nuclear effect on sea quarks and gluons as well as valence quarks. This is the purpose of our investigation. To make a quantitative comparison between the APE model and the $Q^{2}$-rescaling model, some results of these two models are shown in Figs.8-9 in different $x$ scales. Firstly, Figs.89 indicate that both models give reasonable explanations of the EMC effect in the medium $x$ region. However, the expectations of the ratio $R^{C a / D}$ for two different input distributions (GRV and Duke-Owens) are almost the same in the APE model but distinctly different in the $Q^{2}$-rescaling model in which the ratio is slightly sensitive to the choice of free parton distributions. In the lager $x$ region, there are distinct modifications of the recombination effect in the ratio of $R^{C a / D}$ which are similar to, but different from the contributions of the nuclear Fermi motion (cf. Fig.3). For the small $x$ region, we are always seeking for some mechanisms which account for the nuclear shadowing effect. Unfortunately, neither the APE model nor the $Q^{2}$-rescaling model can give suitable explanation of the nuclear shadowing effect. It has been emphasized by Ref.[6] that the shadowing due to the recombinations in the nuclear environment is mainly produced as a result of the modification in the gluon distribution. Considering this recombination effect, both the $Q^{2}$ rescaling model (see Ref. [6]) and the APE model (see Fig.4) can fairly explain the EMC and NMC shadowing data by using the KMRS-B0 input distributions. But, by using harder input gluon distributions such as those of the Duke-Owens(2) [14], the theoretical results (see Figs.8-9) of these two models underestimate the shadowing. It seems, however, that by considering the recombination effect, the prediction of nuclear shadowing in the APE model is closer to the experimental data than that in the $Q^{2}$-rescaling model with these harder input gluon distributions. This is possibly attributed to the better descriptions of the nuclear effect on sea quarks and gluons in our 
model.

As for the prediction of the nuclear Drell-Yan ratio, the result in Fig.6 indicates that, with the same input parton distributions of GRV, the APE model has almost the same prediction of the nuclear Drell-Yan ratio for ${ }^{40} \mathrm{Ca}$ as the $Q^{2}$-rescaling model in the range $0.2<x<0.35$ although there is a slight difference in the nuclear shadowing region $(x<0.1)$. The results of the nuclear Drell-Yan ratios for other nuclei such as $\mathrm{C}, \mathrm{Fe}, \mathrm{W}$ are not presented since the results are qualitatively similar. The results of these nuclear DrellYan ratios do not show a distinct nuclear dependence, which is consistent with the experimental fact.

In summary, we have put forward the APE model which attempts to explain the nuclear effect on the parton distributions by the change of the transverse momentum of partons in the nuclear environment instead of the $Q^{2}$-rescaling model. By means of the APE model with the parton recombination effect, we investigated the nuclear effect including the nuclear structure function $F_{2}^{A}\left(x, Q^{2}\right)$ in the whole $x$ region, nuclear Drell-Yan process and nuclear gluon distribution. The results of the nuclear structure function, in the small $x$ region $(x<0.06)$, are of a little $Q^{2}$-dependence but are very sensitive to the input sea-quark and gluon distributions. By using the proper input parton distributions, we obtained reasonably good agreements with the experimental data of the EMC effect, nuclear Drell-Yan ratio and nuclear gluon distributions.

In addition, the comparison between the APE model and the $Q^{2}$-rescaling model shows, on one hand, that the results are qualitatively similar since they have the same physics origin; and on the other hand, with better description of the nuclear effect on sea quarks and gluons, the value of $R^{A / D}$ in the APE model is less sensitive to the choice of the free parton distributions than that of the $Q^{2}$-rescaling model. Our present investigation should be considered as the first step for seeking for more subtle mechanism with the same physics origin as the $Q^{2}$-rescaling model but with less uncertainties. Further, we are looking forward to a unified description of the nuclear effect and the nuclear shadowing within the framework of the APE model.

\section{ACKNOWLEDGMENTS}

This work was supported by National Natural Science Foundation of China and Natural Science Foundation of Jiangsu Province of China. One of the authors (J.J.Y) would like to thank Dr. S. Kumano for his correspondence about the A-P equation subroutine, and Dr. Vogt for sending their 
computer programs for calculating the parton distributions in Ref. [13]. He also expresses thanks to Prof. H. Q. Shen and Dr. S. A. Aruna for helpful discussions.

\section{References}

[1] EMC, J. J. Aubert et al., Phys. Lett., 123B(1983)275.

[2] NMC, P. Amaudruz et al., Z. Phys. C51(1991)387.

[3] EMC, M. Arneodo et al., Nucl. Phys. B333(1990)1; Phys. Lett. 211B(1988)493.

[4] R.P. Bickerstaff, M.C. Birse and G.A. Miller, Phys. Rev. D33(1986)3228.

[5] NM Collab., P. Amaudruz et al., Nucl. Phys. B371(1992)553.

[6] S. Kumano, Phys. Rev. C48(1993)2016; Phys. Lett. 298B(1993)171.

[7] F. E. Close, R. G. Roberts, and G. G. Ross, Phys. Lett. 129B(1983)346; Nucl. Phys. B296(1988)582; F. E. Close, R. L. Jaffe, R. G. Roberts and G. G. Ross, Phys. Rev. D31(1985)1004.

[8] M.Glück and E. Reya, Nucl. Phys. B145(1978)24.

[9] H. Georgi and H. D. Politzer, Phys. Rev. Lett. 36(1976)1281. (E:37(1976)68); Phys. Rev. D14(1976)1829.

[10] R. Barbieri, J. Ellis, M. K. Gaillard and G. G. Ross, Phys. Lett. 64B(1976)171; Nucl. Phys. B117(1976)50.

[11] G. Altarelli and G. Parisi, Nucl. Phys. B126(1977)298.

[12] J. Kwiecinski, A. D. Martin, W. J. Stirling, and R. G. Roberts, Phys. Rev. D42(1990)3645.

[13] M. Glück, E. Reya and A. Vogt, Z.Phys. C67(1995)433.

[14] D. W. Duke and J. F. Owens, Phys. Rev. D30(1984)49. 
[15] F. E. Close, J. Qiu, and R. G. Roberts, Phys. Rev. D40(1989)2820.

[16] A. H. Mueller and J. Qiu, Nucl. Phys. B268(1986)427; J. Qiu, ibid, B291(1987)746; E. L. Berger and J. Qiu, Phys. Lett. 206B(1988)141.

[17] R. C. Barrett and D. F. Jackson, Nuclear Sizes and Structure (Clarendon, Oxford, 1977).

[18] S. Kumano and J. T. Londergan, Comput. Phys. Commun. 69(1992)373.

[19] G. L. Li, K. F. Liu and G. E. Brown, Phys. Lett. 213B(1988)531.

[20] G. L. Li, Z. J. Cao and C. S. Zhong, Nucl. Phys. A509(1990)757.

[21] L. L. Frankfurt and M. I. Strikman, Phys. Lett. 76B(1978)333.

[22] A.W Hendry, D. B. Lichtenberg and E. Predazzi, Nuovo Cimento 92A(1986) 427 .

[23] G. E. Brown and M. Rho, Phys. Lett. 237B(1990)3.

[24] D. M. Alde et al., Phys. Rev. Lett. 64(1990)2479.

[25] E. L. Berger and D. Jones, Phys. Rev. D23(1981)1521; A. D. Martin, C. K. Ng and W. J. Stirling, Phys. Lett. 191B(1987)200.

[26] P. Braun-Munzinger and G. David, in: Proc. Intern. Workshop on Gross properties of nuclei and nuclear excitation (Hirschegg, Austria, January 1992) ed. H.Feldmeier. 


\section{Figure Captions}

Fig.1. Deep inelastic scattering in the free parton model.

Fig.2. Momentum cutoff for leak-out partons, $\beta(x)=\exp \left(-\frac{m_{N}^{2} y_{0}^{2} x^{2}}{2}\right)$.

Fig.3. Comparisons with SLAC data for ${ }^{40} \mathrm{Ca}$ with Ferim motion correction. Input parton distributions are those of KMRS-B0 [12]. $Q^{2}=4.0 \mathrm{GeV}^{2}$.

Fig.4(a-b). Comparisons of the ratio $R^{C a / D}$ with EMC-90 [3] and NMC [2] data by using (a) the GRV 13 input and (b) the KMRS-B0 [12 input. $Q^{2}=4.0 \mathrm{GeV}^{2}$ and $8.0 \mathrm{GeV}^{2}$ for the solid and dashed curves, respectively.

Fig.5. Comparisons of the ratio $R^{C a / D}$ with EMC-90 [3] and NMC [2] data by using different input distributions. The solid, dashed and dotted curves are for the KMRS-B0 [12], the GRV [13] and Duke-Owens(2) [14, respectively. $Q^{2}=4.0 \mathrm{GeV}^{2}$.

Fig.6. The nuclear Drell-Yan ratios $T^{C a / D}(x)$ predicted by the present model(solid curve) and the $Q^{2}$-rescaling model(dashed curve) are plotted versus x by using the GRV input distributions at $Q^{2}=20 \mathrm{GeV}^{2}$. The experimental data are taken from the E772 Collaboration [24].

Fig.7. Comparisons of the result of $G_{S n}(x) / G_{C}(x)$ with NMC data [0] by using the KMRS-B0 input distributions at $Q^{2}=4 \mathrm{GeV}^{2}$.

Fig.8(a-b). The ratio $R^{C a / D}(x)$ given by the APE model is plotted. (a) in the linear x scale; (b) in the logarithmic x scale. Results by using the GRV inputs(solid curve) and the Duke-Owens(2) inputs(dashed curve) at $Q^{2}=4$ $\mathrm{GeV}^{2}$.

Fig.9(a-b). The ratio $R^{C a / D}(x)$ given by the $Q^{2}$-rescaling model is plotted. (a) in the linear x scale; (b) in the logarithmic x scale. Results by using the GRV inputs(solid curve) and the Duke-Owens(2) inputs(dashed curve) at $Q^{2}=4 \mathrm{GeV}^{2}$. 


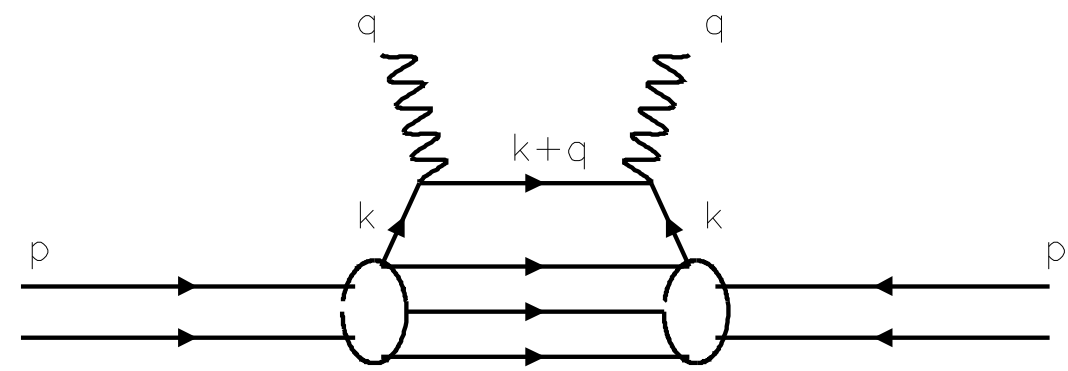

Fig. 1 


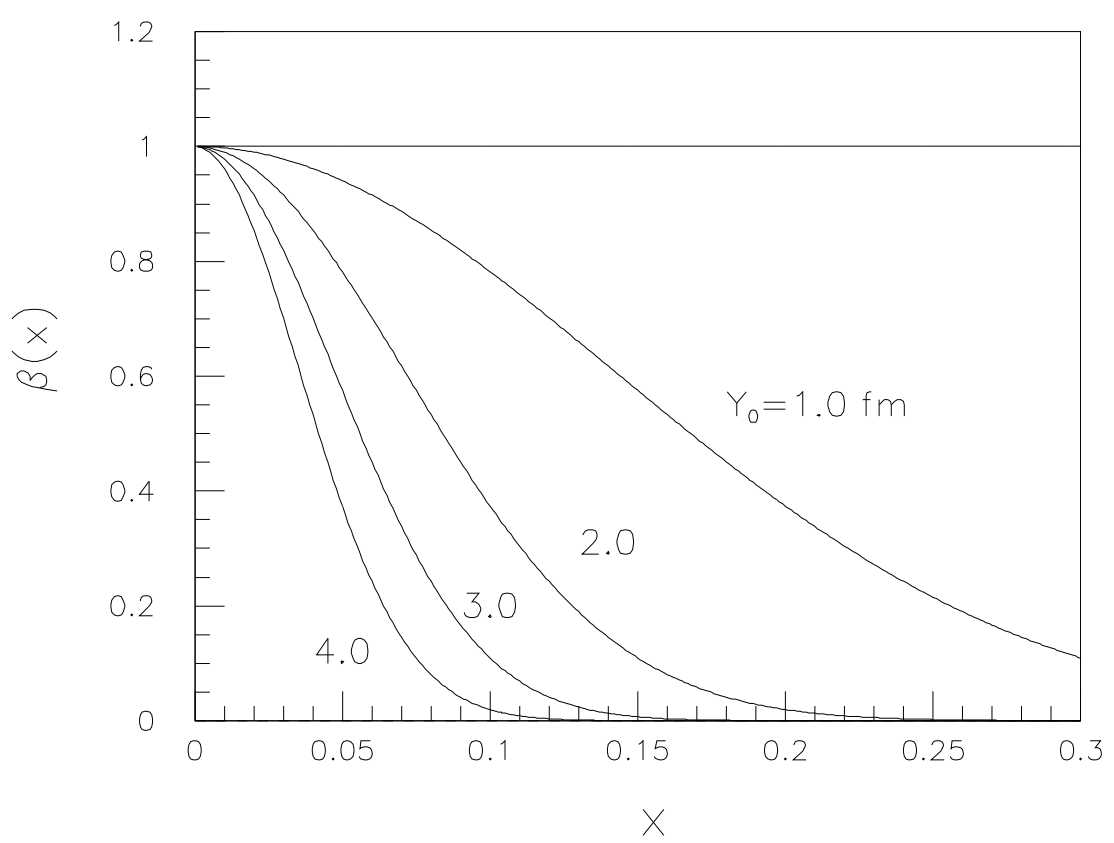

Fig. 2 


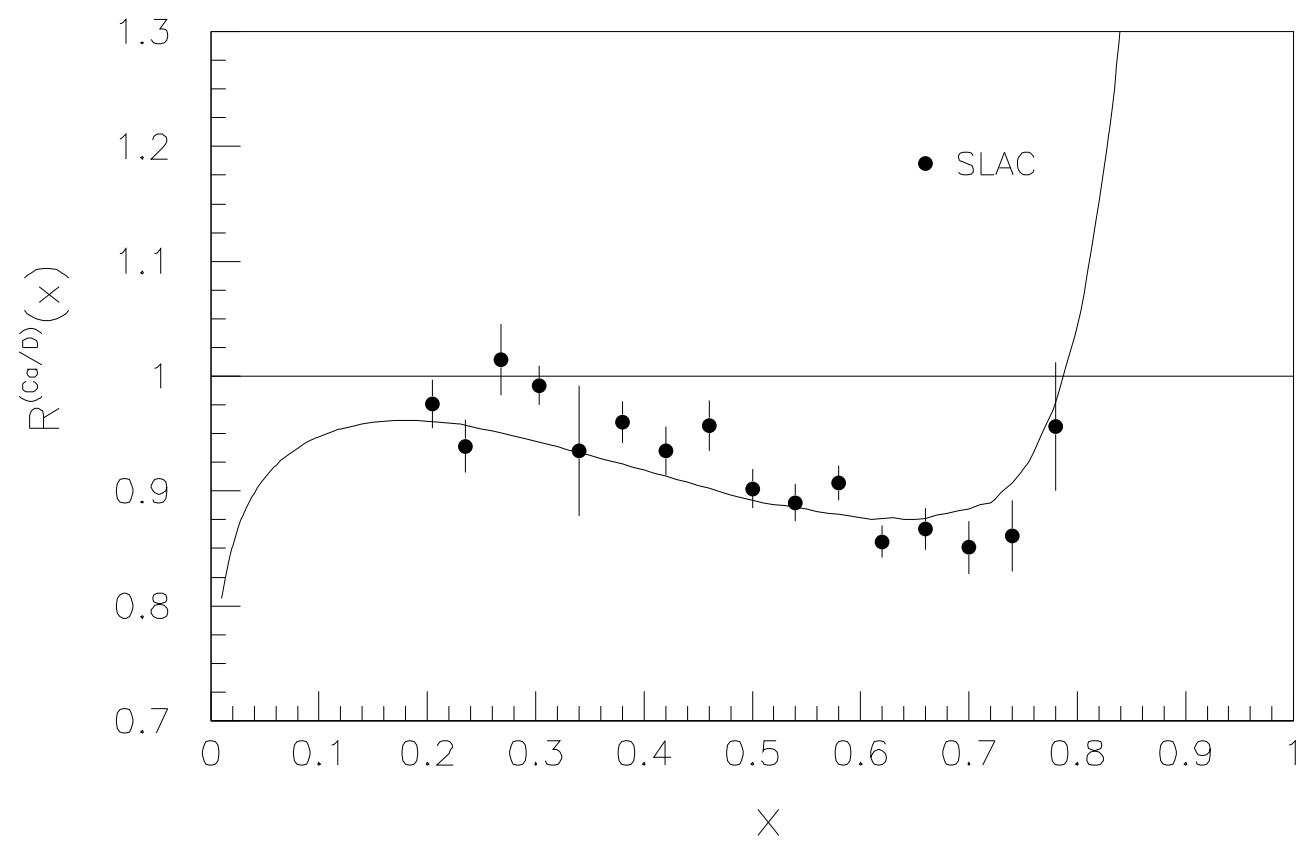

Fig.3 


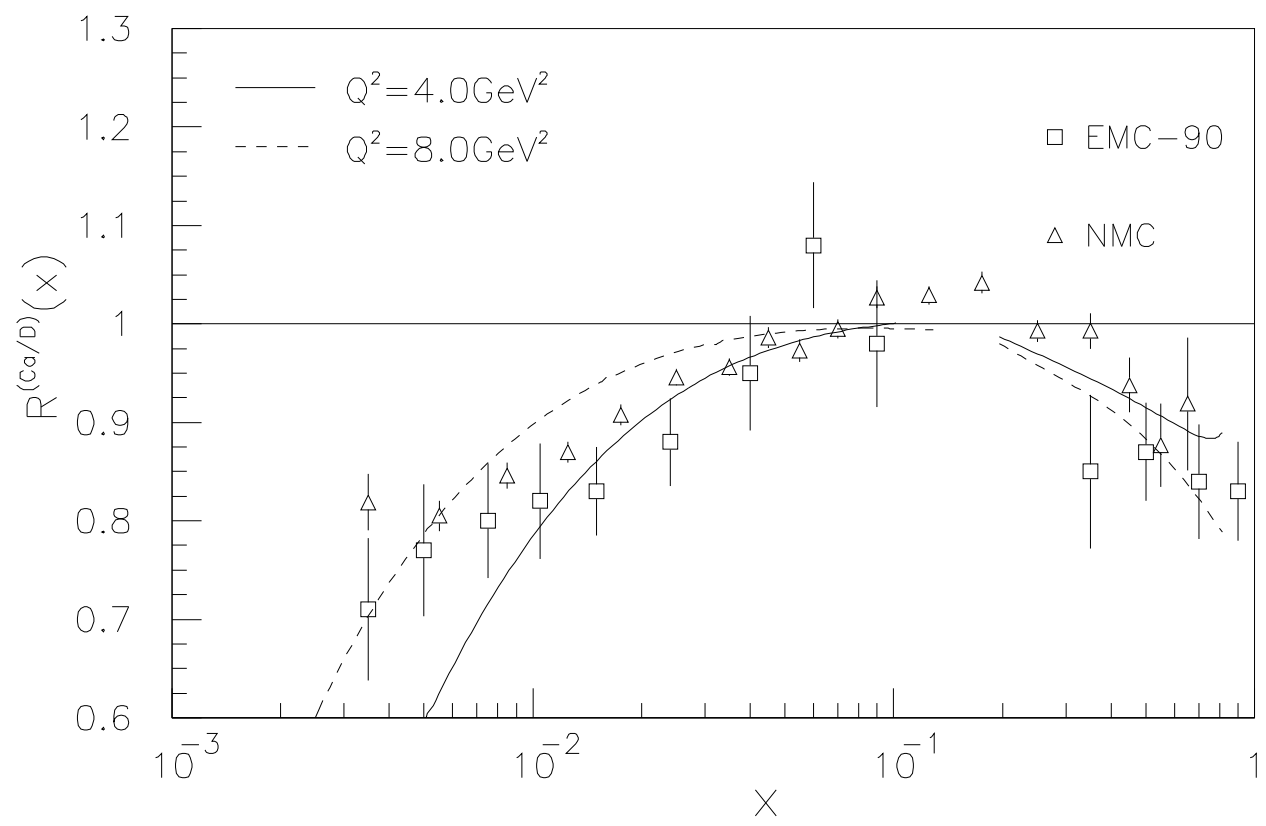

Fig.4(a) 


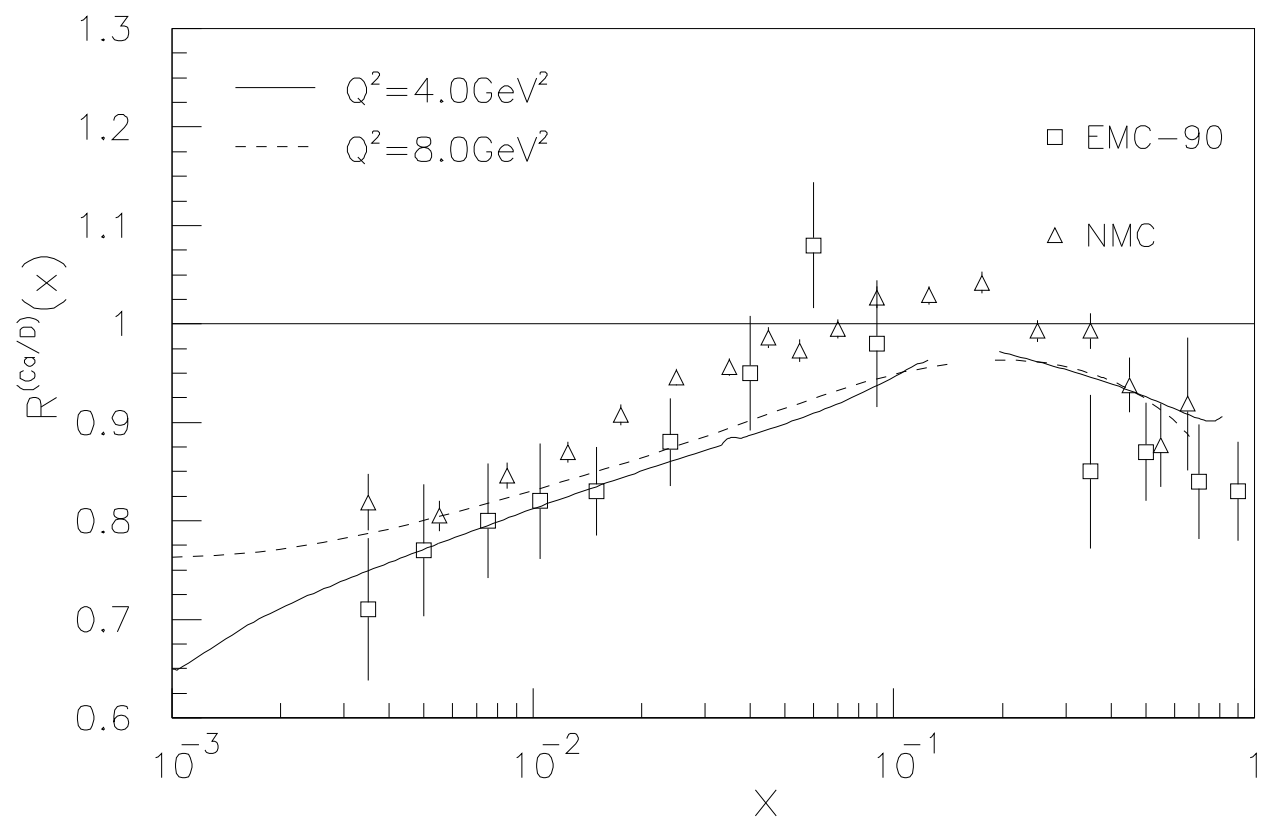

Fig.4(b) 


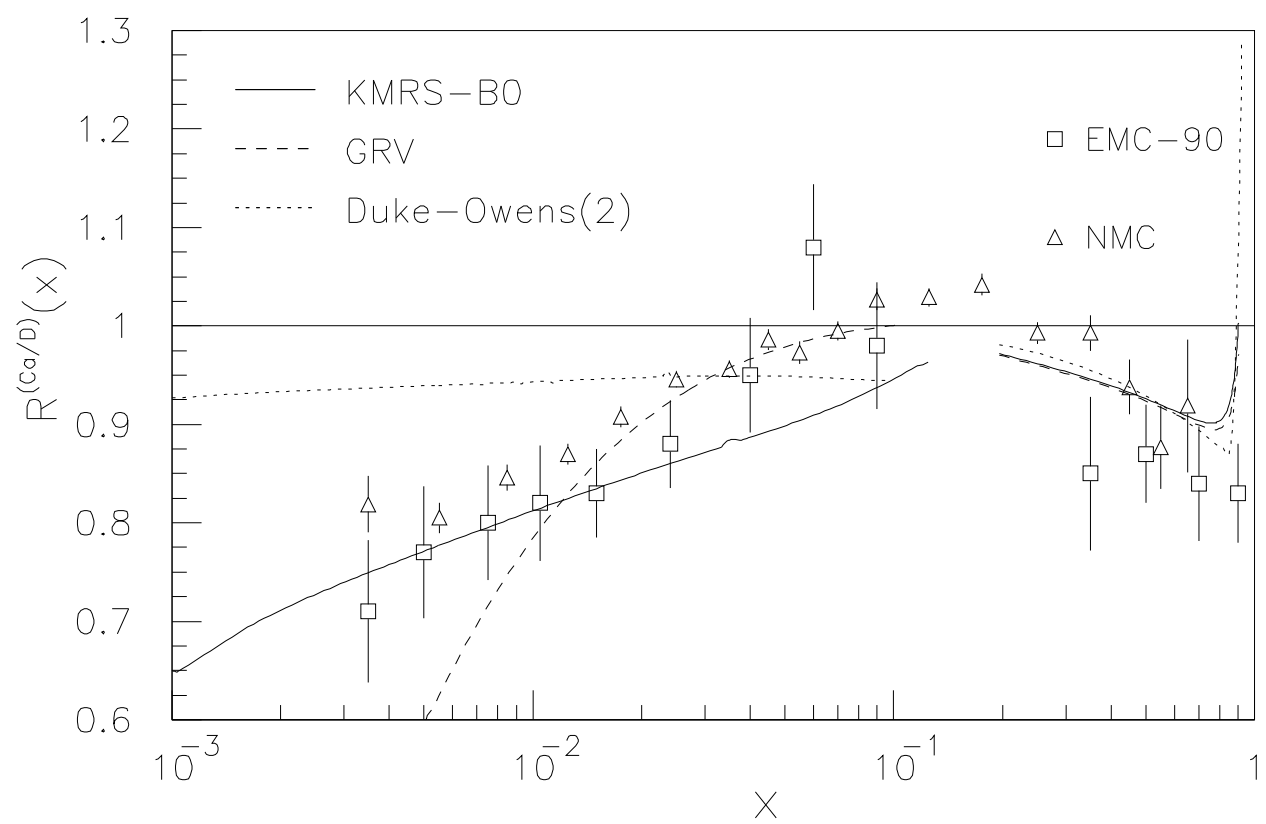

Fig. 5 


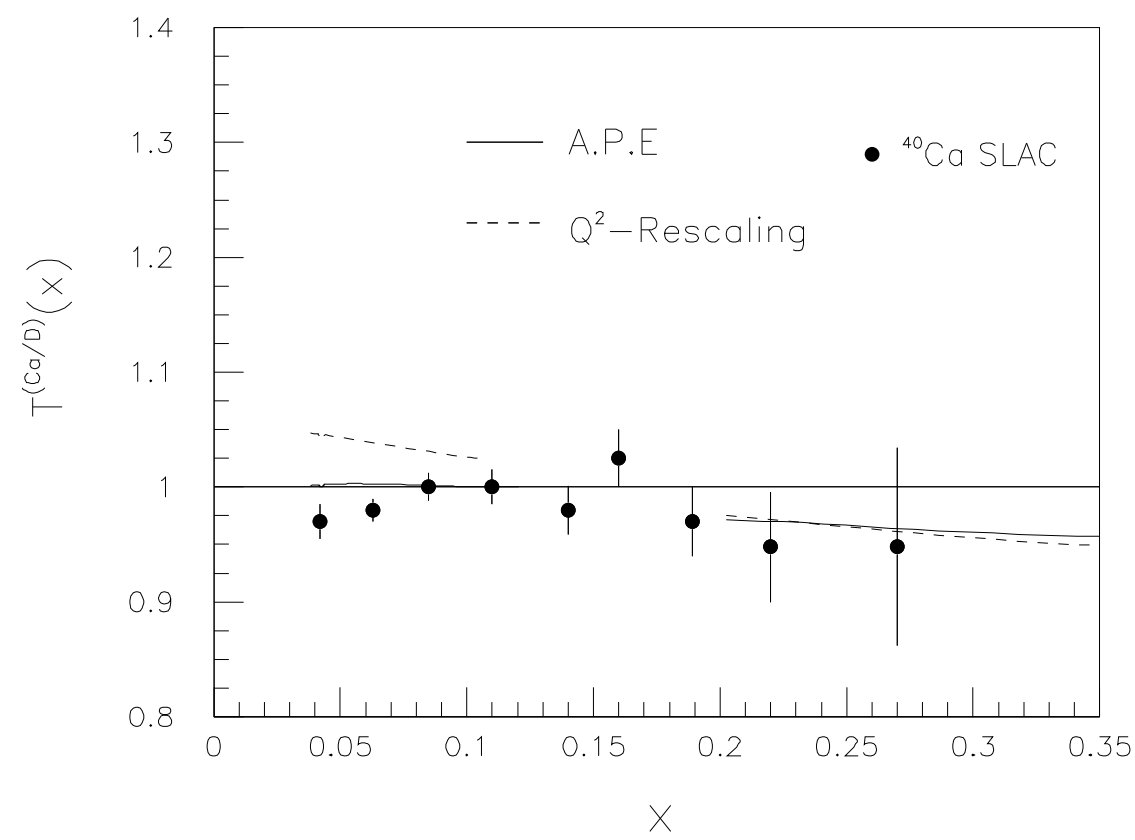

Fig.6 


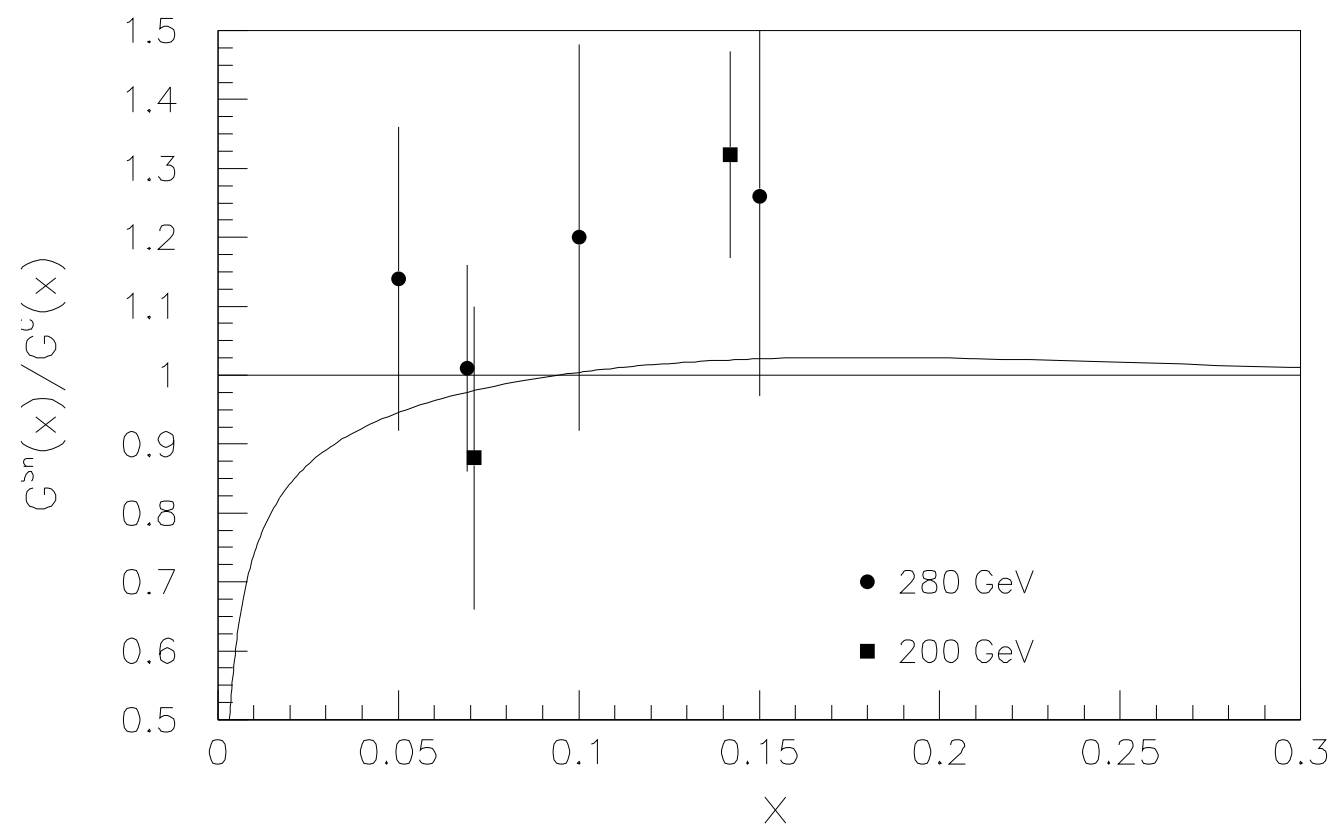

Fig.7 


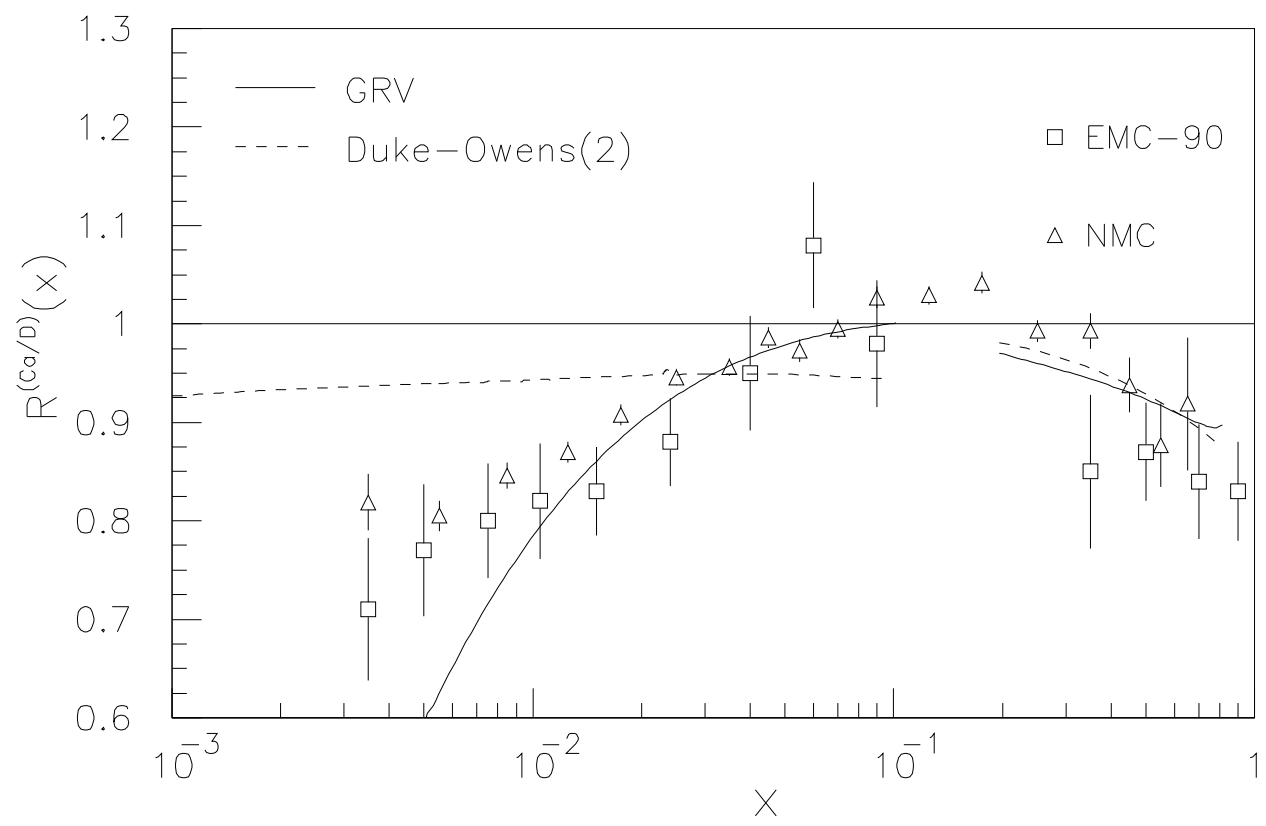

Fig.8(a) 


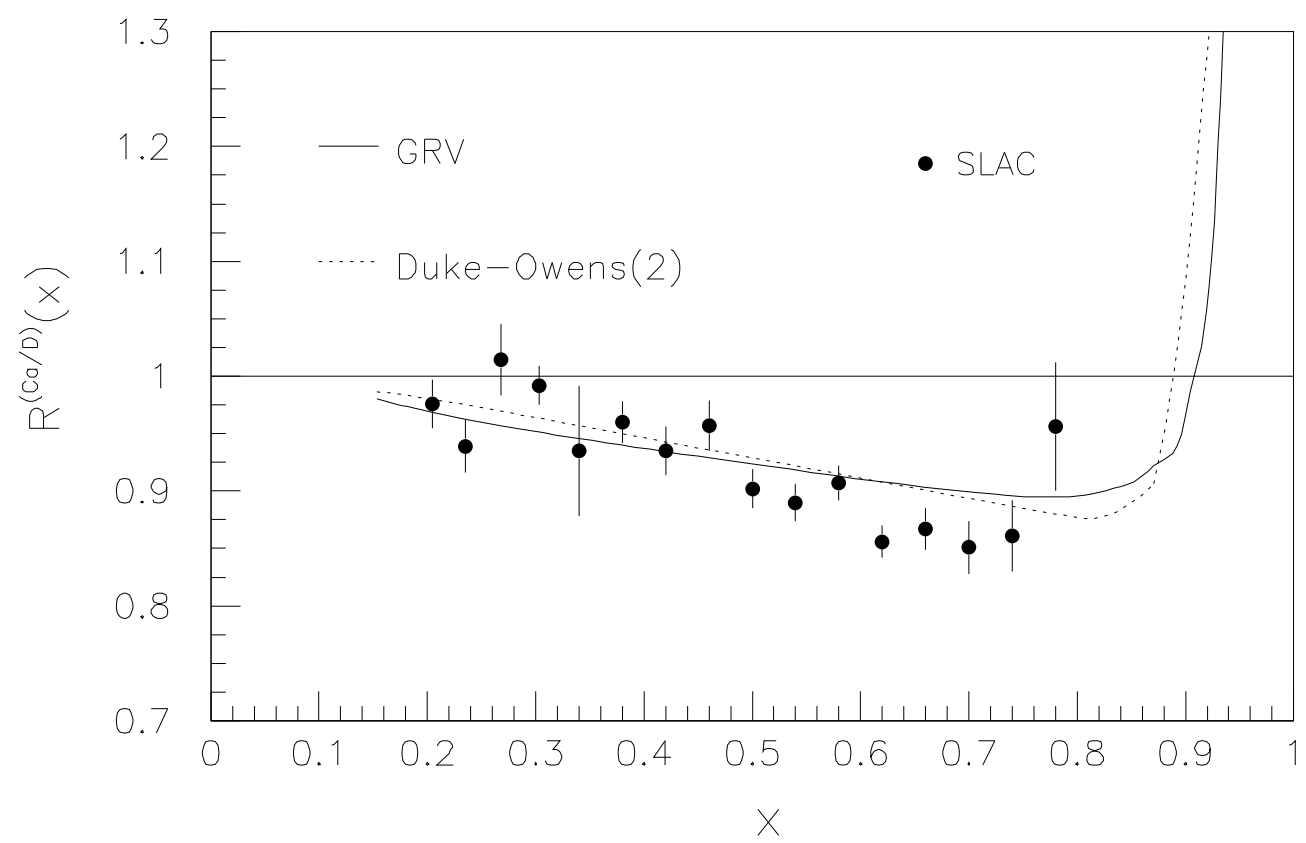

Fig.8(b) 


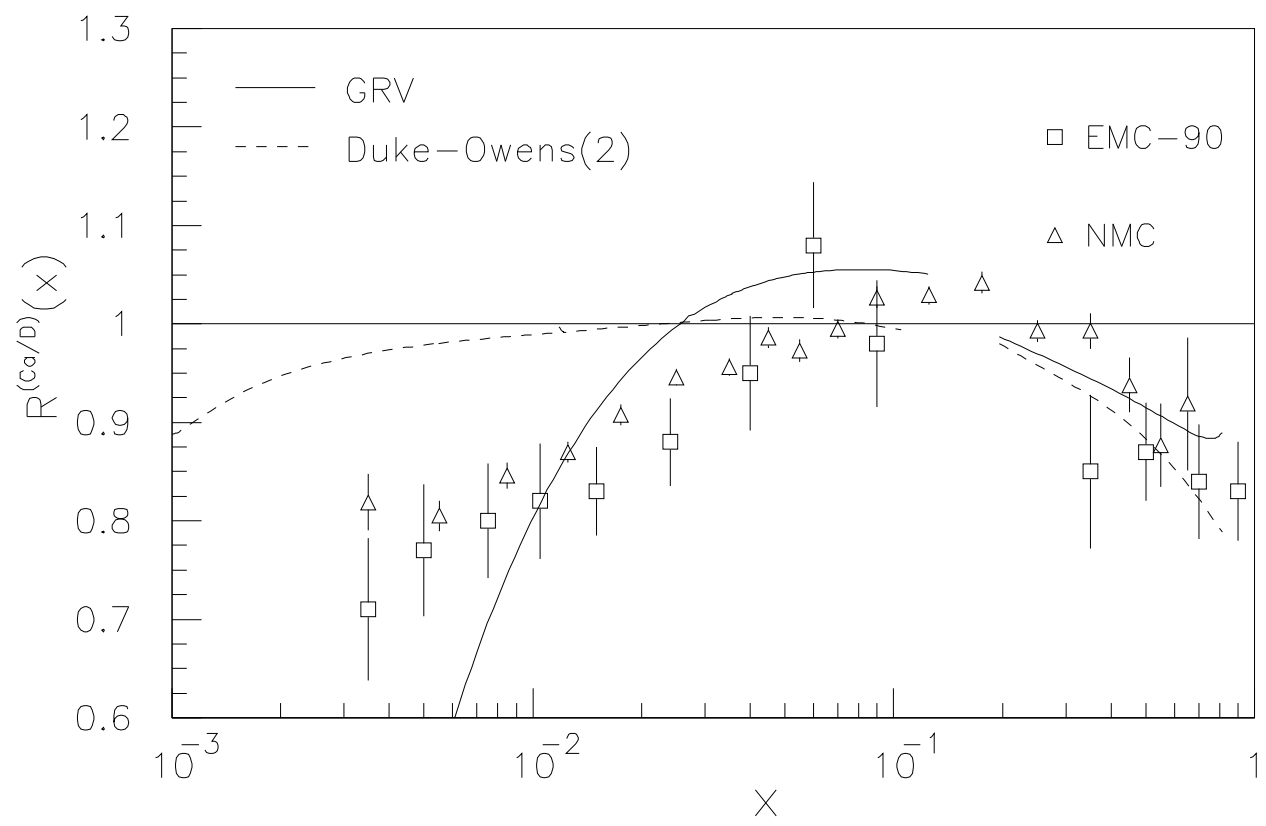

Fig.9(a) 


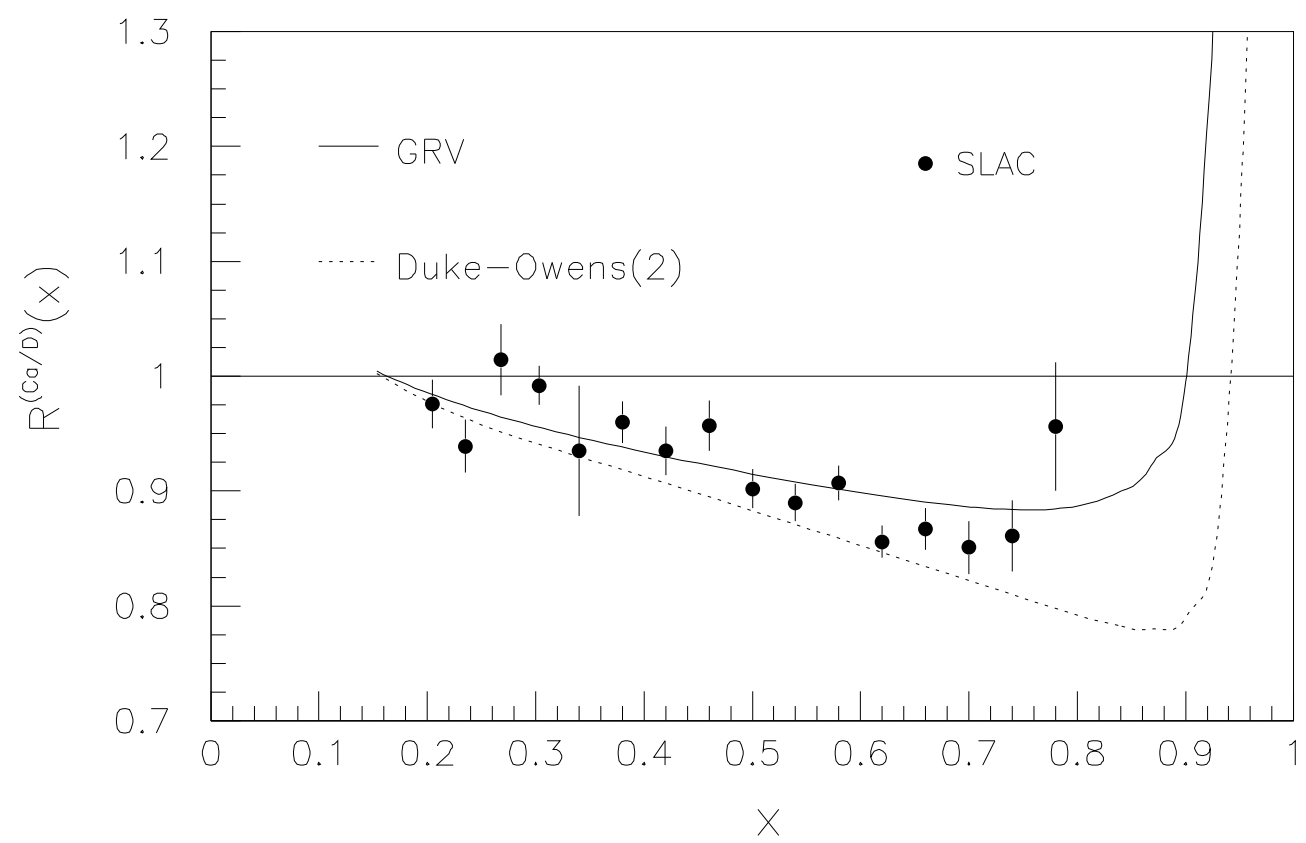

Fig.9(b) 